\title{
The role of myokines in muscle health and disease
}

\author{
Adam P. Lightfoot, Robert G. Cooper \\ MRC Arthritis Research UK Centre for Integrated Research into Musculoskeletal Ageing, Department of \\ Musculoskeletal Biology, Institute of Ageing and Chronic Disease, University of Liverpool, UK. \\ Corresponding Author: Professor Robert G. Cooper, MD, FRCP Department of Musculoskeletal Biology \\ Institute of Ageing and Chronic Disease, Faculty of Health \& Life Sciences, University of Liverpool, William \\ Duncan Building, West Derby Street, Liverpool, L7 8TX. robert.cooper@liverpool.ac.uk
}


Purpose of review: To update on the concept that muscle-derived cytokines (myokines) play important roles in muscle health and disease

Recent findings: IL-6 is released from normal skeletal muscle in response to exercise, mediating both antiinflammatory responses and metabolic adaptations, actions contradictory to the prevailing view that IL-6 is a proinflammatory cytokine, i.e. inducing and propagating disease. The anti-inflammatory effects of IL-6 result from its trans-membrane signalling capability, via membrane-bound receptors, while its pro-inflammatory effects result instead from signalling via the soluble IL-6 receptor and gp130. IL-15 is elevated following exercise, promoting muscle fibre hypertrophy in some circumstances, while inducing fibre apoptosis in others. This functional divergence appears due to variations in expression of IL-15 receptor isoforms. Decorin, a recently described myokine, is also elevated following exercise in normal muscle, and promotes muscle fibre hypertrophy by competitively binding to, and thus inhibiting, myostatin, a negative regulator of muscle protein synthesis. Exercise-induced myostatin downregulation thus promotes muscle fibre growth, prompting recent trials of a biological myostatin inhibitor in inclusion body myositis (IBM).

Summary: Myokines appear to exert diverse beneficial effects, though their mechanistic roles in myositis and other myopathologies remain poorly understood.

Keywords: myositis, myokines, skeletal muscle, cytokines, IIM

\section{Introduction}

When contemplating that skeletal muscle possesses paracrine and endocrine capabilities, it is important to consider the role of secreted factors across cells and tissues in general. Approximately 10-15\% of all proteins encoded by the human genome are targeted for secretion [1], and secreted proteins provide the key mechanisms to enable intercellular communications and dynamic processes, such as differentiation and proliferation [2]. The profile of secreted proteins from cells is highly variable, and may change according to prevailing stimuli, a process which has been implicated in the pathogenesis of a range of diseases; emphasising the biological importance of factors secreted from all cells and tissues [3].

Given that skeletal muscle accounts for $\sim 40 \%$ of total body protein, it seems reasonable to suggest that musclederived protein secretions likely make a significant contribution to overall circulating levels of detectable proteins. That muscle fibres intrinsically express cytokines was first demonstrated by the increased skeletal muscle expression of interleukin-6 (IL-6) detected in response to exercise [4]. In vitro demonstration of elevated 
expression of muscle IL-6, transforming growth factor beta (TGF- $\beta$ ) and granulocyte macrophage colony stimulating factor (GM-CSF) have also been shown in response to pro-inflammatory stimuli [5]. Since then, the repertoire of detectable myokines has steadily grown [6]. With technological advances in protein identification, using a combination of in vitro, ex vivo and in silico approaches, a diverse skeletal muscle "secretome" has become increasingly apparent [7-10]. The gambit of myokines released from skeletal muscle is considerable, and implicated in mediating cross-talk and between-cell signalling in various tissues and organs. This article focuses on recent developments in relation to muscle-derived cytokines (myokines), commenting on their potential contribution in health and in the wider rheumatological context, and proposing specific roles for myokines in the pathogenesis of myositis

\section{From cytokines to myokines}

IL-6: The main focus of skeletal muscle IL-6 research has centred on its production from normal muscle in response to exercise [4], following which there is an increase in IL-6 gene expression, a process mediated by calcium ions and the calcineurin pathway [11, 12]. Significantly increased circulating levels of IL-6 are also reported following bouts of acute exercise, a process which appears independent of local muscle fibre damage [13]. Alongside these local and systemic exercise-induced IL-6 elevations, coincidental increases in levels of the anti-inflammatory cytokines IL-1ra and IL-10 also occur [14]. That muscle produces and releases IL-6 is further supported by in vitro studies, in which IL-6 release can be induced in muscle in response to pro-inflammatory stimuli (i.e. by IL-1 $\alpha / \beta$, TNF- $\alpha$ and IFN- $\gamma$ ), a process mediated in part by reactive oxygen species, specifically mitochondrial superoxide and the transcription factor nuclear factor kappa B (NFKB) (Figure 1) [5, 15-17]. Further mechanistic research has suggested that the basal expression and secretion of skeletal muscle IL-6 is controlled by an intrinsic (within muscle) self-sustaining circadian rhythm. Interestingly, disruption of the latter, using short interfering RNA techniques, causes a downregulation of IL-6 secretion [18].

The anti-inflammatory effects of IL- 6 are suggested by the observation that infusions of recombinant IL- 6 can reduce the size of endotoxin-induced elevations of circulating TNF-alpha levels [19]. Similarly, a recent study of macrophages reported that IL- 6 can suppress their pro-inflammatory responses to endotoxin, this effect being mediated by interactions with IL-4 and its receptor. Collectively these data suggest that IL-6 can act as an antiinflammatory myokine [20]. However, this exercise-associated anti-inflammatory function of IL-6 appears at odds with the widely accepted view that IL-6 is primarily a pro-inflammatory cytokine [21]. Moreover, in the context of skeletal muscle, elevated levels of circulating IL-6 are viewed as a catabolic and so atrophic stimulus for muscle [22]. A secondary function of muscle-derived IL-6 is its important role in energy regulation and metabolism: i.e. 
exercise-induced IL-6 release from muscle appears inextricably linked to muscle glycogen levels [23], thus increasing muscle glucose uptake, insulin secretion and promoting fatty acid oxidation [24]. There is clearly a disparate impact of IL-6 in terms of pro- or anti-inflammatory functions, which in turn appear dependent on its interaction with receptors, a process termed trans-signalling [25]. This suggests that IL-6 pro-inflammatory functions are activated when signalling is via the soluble IL-6 receptor and its interaction with the membranebound gp130 protein subunit. In contrast, the anti-inflammatory functions of IL-6 appear to be activated when signalling is via membrane bound IL-6 receptors [25]. Overall, the impact and paracrine and endocrine mechanisms of action of IL-6 on muscle are complex and diverse, so further mechanistic interrogation is needed. IL-8: IL-8 is a neutrophil chemoattractant mediating angiogenesis, and exercise is known to induce significant increases in its gene expression levels within normal skeletal muscles [26], while elevations in circulating levels of IL-8 appear to be associated with damaging exercise regimes, for instance eccentric (i.e lengthening) contractions. It seems likely that exercise-induced intramuscular IL-8 changes are associated with fibre damage, the latter more likely during eccentric contractions, while elevated circulating levels are more likely derived from intramuscular immune cell infiltrations occurring secondary to muscle damage. Functionally, our knowledge of the impact of IL-8 on skeletal muscle is relatively sparse. However, IL-8 has been shown to interface with the CXCR2 receptor, which is expressed in muscle and endothelial cells, and which is also associated with the promotion of angiogenesis [27]. Based on this, it seems reasonable to suggest that IL-8 may play an important role in mediating beneficial muscle cellular adaptations in response to exercise.

IL-15: IL-15 is a pro-inflammatory cytokine which has the capacity to activate both B- and T-cells [28]. Moreover, it can stimulate the expression and secretion of inflammatory cytokines such as TNF-alpha in monocytes [29]. The role of IL-15 in the context of skeletal muscle was first described some 20 years ago, when it was reported to significantly promote anabolism, and then when used as a potential anabolic agent to treat certain muscle wasting disorders $[30,31]$. Interestingly, more recent research has shown that resistance exercise has the potential to induce increases in normal skeletal muscle IL-15 gene expression levels [32]. Also, intravenous IL-15 administration in a rodent cachexia model is associated with a reduction in white adipose tissue deposition, and a significant protective effect against loss of muscle mass $[33,34]$. Moreover, osmotic pump delivery of IL-15 has been shown to improve muscle function in the $m d x$ mouse model of Duchenne muscular dystrophy [35]. In contrast, administration of exogenous IL-15 to both young and ageing wild-type mice does not induce skeletal muscle hypertrophy, but instead promotes apoptosis [36]. This functional discordance is thought to be associated with variations in the interactions of IL-15 with its receptors. Studies in a murine model with the IL-15 alpha receptor 
(IL-15Ralpha) knocked out in all tissues, showed altered physiological properties, with affected mice displaying increased exercise capacity and resistance to fatigue [37]. This effect was further emphasised when examining IL15Ralpha gene SNPs, whereby the presence of these SNPs was positively associated with good performances in human athletes competing in endurance events [37]. Thus, SNPs in the IL-15 receptor isoform genes may dictate the response to elevations in IL-15, to either promote hypertrophy, apoptosis or adaptations to exercise training. Further investigations into IL-15 receptor isoforms and potential gene SNPs are clearly indicated.

CCL and CXCL family: Chemokines can be categorised into CC and CXC subtypes. They are small peptides, whose primary function is to provoke chemotaxis. Members of the chemokine sub-families have a chemotactic affinity for specific cell types (e.g. T-cells or monocytes or neutrophils etc). In terms of the CCL chemokines, proteomic analyses of skeletal muscle have identified expression of the CCL2, CCL7 and CCL8 subtypes [38]. Furthermore, examination of normal muscle cells in vitro confirms that CCL2 and CCL5 are secreted directly in response to a TNF- $\alpha$ challenge [17]. Overexpression of MHC I in muscle cells in vitro has been demonstrated to drive CCL2 and CCL5 release via the ER stress pathway [39]. The overall function of CCL chemokines in the context of normal muscle physiology is poorly understood, although CCL2 has been reported to play an integral role in muscle fibre regeneration post injury [40]. Moreover, CCL4 has in vitro been shown to be released from muscles overexpressing MHC I [39]. A role has also been reported for CCL4 in muscle regeneration, whereby knockdown of the receptor CXCR2, which interacts with CCL4, results in impaired muscle repair [40]. In terms of CXC chemokines, CXCL1 (a neutrophil chemoattractant) has been reported to be secreted from muscle cells in vitro following stimulation with TNF- $\alpha$, and in response to overexpression of MHC I [17, 39].

Decorin: Decorin is a leucine rich proteoglycan and is a crucial component of the extracellular matrix and a newly characterised myokine. Recent research has indicated that decorin is secreted from muscle into the circulation following acute bouts of resistance exercise. In terms of its function, decorin has been shown in transgenic models to induce the upregulation of factors associated with myogenesis, specifically, elevations in MyoD1, follistatin and Mighty, and followed by a down regulation in Atrogin-1 and MuRF-1, i.e markers or promotors of muscle atrophy. Mechanistically, secreted decorin binds extracellularly to myostatin (Figure 1). As the negative regulatory effects of myostatin are then inhibited, this induces myofibre hypertrophy [41]. These findings clearly suggest that decorin may be an important mediator of the beneficial impact of, and adaptations to, exercise training.

Myostatin: Myostatin is a member of the TGF- $\beta$ super family, and a potent negative regulator of muscle mass

(Figure 1). In normal, physiological conditions, myostatin interacts with and binds to the activin receptor 
complex, resulting in a signalling cascade which acts to prevent unopposed activin-induced muscle hypertrophy. Myostatin expression is downregulated in response to most types of exercise, thus promoting protein synthesis. In contrast to the vast majority of other myokines, where circulating levels are elevated in response to exercise, serum myostatin levels fall with exercise. It is likely that this exercise induced down-regulation forms part of a beneficial hypertrophic adaptive response. The importance of the activin:myostatin interaction appears clear, and this area has become the focus of significant commercial awareness in the context of developing drugs capable of combating disease-associated muscle atrophy. Such pharmacological interest has led to the development of an antagonist to the activin type II receptor, called Bimagrumab. A recent "proof of concept" trial of this agent in patients with IBM, a disease associated with progressive and refractory muscle wasting, showed potentially beneficial effects, as evidenced by increased quadriceps femoris volumes after 8 weeks of treatment [42].

\section{Could myokines be involved in the pathogenesis of myositis?}

The notion that skeletal muscle may itself be a source of potentially pathogenic muscle-derived cytokines, i.e. over and above those being produced by infiltrating inflammatory cells, provides an interesting perspective on what precisely drives the various pathogenic components of myositis. Historically it has been "accepted" that immunologically targeted inflammatory cell infiltration of the muscle cell membrane is the major pathological cause of muscle fibre damage, and that this process is orchestrated by cytokines and chemokines derived from infiltrating immune cells. However, and given the vast protein source that skeletal muscle represents, it seems reasonable to envisage that muscle may itself have the potential to act as a significant source of cytokines, i.e. myokines, which may contribute to disease pathogenesis. In fact research does suggest that myokines do play pathogenic roles in both inflamed and non-inflamed muscle, and so do contribute to the induction of muscle weakness and dysfunction in myopathies, including in myositis. In inflamed skeletal muscle, cytokines released by infiltrating immune cells likely interact with relevant receptors on the surface of muscle fibres, and thus induce upregulations/downregulations of a range of myokines (see Figure 1). This suggestion is supported by research which shows a range of myositis-relevant cytokines which could induce expression of other cytokines and MHC I in muscle $[5,16,17]$. The resulting expression and release of myokines could then contribute to immune cell chemotaxis or local immune cell activation, or have paracrine signalling effects on neighbouring muscle fibres. Indeed, in treatment-suppressed and non-inflamed myositic muscle, myokines may well play a role in the commonly reported phenomenon of persistent muscle weakness in the absence of inflammation. Here, nonimmune cell mediated mechanisms, such as ER stress pathway activation, are reported to contribute to weakness induction [43, 44]. Also, activation of the ER stress pathway in both muscle and non-muscle systems can 
upregulate the expression of a range of cytokines $[45,46]$. Myokines thus released from muscle, inflamed or not, could then exert deleterious paracrine signalling effects on neighbouring fibres, an important consideration potentially explaining non immune cell mediated weakness induction. In addition, the involvement of myokines may explain reports that weakness can even precede inflammatory cell infiltration, i.e. in a murine myositis model [47], and suggestions that inflammation in myositis may be, at least initially, a "secondary" phenomenon [44].

In health, exercise clearly induces beneficial myokine secretions, and which mediate well described antiinflammatory effects. Recent, state-of-the-art genomic and proteomic research has now demonstrated that (endurance) exercise is also beneficial in PM and DM patients, clearly improving aerobic capacity and suppressing various inflammatory mechanisms present in the muscles of these patients [48]. Overall, these important results demonstrate that exercise in myositis patients is not only safe, but clearly also functionally efficacious, and capable of inducing significant beneficial molecular adaptations. Although myokines were not directly assessed in this recent research, it seems logical to conclude that myokines likely did play a role in mediating the exercise-induced anti-inflammatory adaptive responses demonstrated in myositis patients.

\section{Conclusion}

Components of the skeletal muscle secretome appear responsive to physiological and pathological conditions affecting muscle. Myokines mediate beneficial adaptations related to exercise, but myokine effects vary according to prevailing conditions, and currently there is a paucity of insight regarding what governs myokine effects. Modifications of myokine interactions with their receptors, as occurs in the case of IL-6 and IL-15, may dictate and explain response differences. What governs the role of myokines in pathological circumstances, such as myositis, remains unclear. Further characterisation of the secretome may provide mechanistic insights to facilitate future drug developments in a situation where available therapies remain relatively ineffective [49].

\section{KEY POINTS}

- Myokines represent a diverse range of cytokines and chemokines derived from skeletal muscle.

- Exercise training modifies the myokine profile secreted, thus mediating beneficial local and systemic effects and favourably modulating muscle adaptive responses both in health and disease.

- The favourable myokine responses to exercise appear in contrast to those described in variety of pathological conditions, including myositis. 
- A greater understanding of myokines in the context of health and disease is now needed.

\section{Acknowledgments}

The authors would like to thank University of Liverpool, Myositis UK and The Myositis Association (TMA) for their generous financial support.

\section{Competing interests}

The authors have no competing interests to declare

\section{References and recommended reading}

Papers of particular interest have been highlighted as:

- of special interest

•• of outstanding interest

1. Pavlou MP, Diamandis EP: The cancer cell secretome: a good source for discovering biomarkers? $J$ Proteomics 2010, 73:1896-1906.

2. Karagiannis GS, Pavlou MP, Diamandis EP: Cancer secretomics reveal pathophysiological pathways in cancer molecular oncology. Mol Oncol 2010, 4:496-510.

3. Cox TR, Rumney RM, Schoof EM, Perryman L, Hoye AM, Agrawal A, Bird D, Latif NA, Forrest H, Evans HR, et al: The hypoxic cancer secretome induces pre-metastatic bone lesions through lysyl oxidase. Nature 2015, 522:106-110.

4. Ostrowski K, Rohde T, Zacho M, Asp S, Pedersen BK: Evidence that interleukin-6 is produced in human skeletal muscle during prolonged running. J Physiol 1998, 508 ( Pt 3):949-953.

5. Nagaraju K, Raben N, Merritt G, Loeffler L, Kirk K, Plotz P: A variety of cytokines and immunologically relevant surface molecules are expressed by normal human skeletal muscle cells under proinflammatory stimuli. Clin Exp Immunol 1998, 113:407-414.

6. Bhatnagar S, Panguluri SK, Gupta SK, Dahiya S, Lundy RF, Kumar A: Tumor necrosis factor-alpha regulates distinct molecular pathways and gene networks in cultured skeletal muscle cells. PLoS One 2010, 5:e13262.

7. Yoon JH, Kim J, Song P, Lee TG, Suh PG, Ryu SH: Secretomics for skeletal muscle cells: a discovery of novel regulators? Advances in biological regulation 2012, 52:340-350.

8. Bortoluzzi S, Scannapieco P, Cestaro A, Danieli GA, Schiaffino S: Computational reconstruction of the human skeletal muscle secretome. Proteins 2006, 62:776-792.

9. Henningsen J, Rigbolt KT, Blagoev B, Pedersen BK, Kratchmarova I: Dynamics of the skeletal muscle secretome during myoblast differentiation. Mol Cell Proteomics 2010.

- 10. Hartwig S, Raschke S, Knebel B, Scheler M, Irmler M, Passlack W, Muller S, Hanisch FG, Franz T, Li X, et al: Secretome profiling of primary human skeletal muscle cells. Biochim Biophys Acta 2014, 1844:1011-1017.

A study using sensitive proteomics to idenitfy and characterise components of the skeletal muscle secretome.

11. Luo G, Hershko DD, Robb BW, Wray CJ, Hasselgren PO: IL-1beta stimulates IL-6 production in cultured skeletal muscle cells through activation of MAP kinase signaling pathway and NF-kappa B. Am J Physiol Regul Integr Comp Physiol 2003, 284:R1249-1254.

12. Weigert C, Dufer M, Simon P, Debre E, Runge H, Brodbeck K, Haring HU, Schleicher ED: Upregulation of IL-6 mRNA by IL-6 in skeletal muscle cells: role of IL-6 mRNA stabilization and Ca2+-dependent mechanisms. Am J Physiol Cell Physiol 2007, 293:C1139-1147. 
13. Peake JM, Suzuki K, Hordern M, Wilson G, Nosaka K, Coombes JS: Plasma cytokine changes in relation to exercise intensity and muscle damage. Eur J Appl Physiol 2005, 95:514-521.

14. Steensberg A, Fischer CP, Keller C, Moller K, Pedersen BK: IL-6 enhances plasma IL-1ra, IL-10, and cortisol in humans. Am J Physiol Endocrinol Metab 2003, 285:E433-437.

15. Kosmidou I, Vassilakopoulos T, Xagorari A, Zakynthinos S, Papapetropoulos A, Roussos C: Production of interleukin-6 by skeletal myotubes: role of reactive oxygen species. Am J Respir Cell Mol Biol 2002, 26:587-593.

16. Chevrel G, Granet C, Miossec P: Contribution of tumour necrosis factor alpha and interleukin (IL) 1beta to IL6 production, NF-kappaB nuclear translocation, and class I MHC expression in muscle cells: in vitro regulation with specific cytokine inhibitors. Ann Rheum Dis 2005, 64:1257-1262.

-17. Lightfoot AP, Sakellariou, G.K, Nye, G.A, McArdle, F, Jackson, M.J, Griffiths, R.D, McArdle, A: SS31 attenuates TNF- $\alpha$ induced cytokine release from C2C12 myotubes. Redox Biology 2015, 10:253-259.

This study idenitified a specific type of ROS, i.e mitochondrial superoxide, as a mediator of myokine release in response to inflammatory stimuli.

•18. Perrin L, Loizides-Mangold U, Skarupelova S, Pulimeno P, Chanon S, Robert M, Bouzakri K, Modoux C, Roux-Lombard P, Vidal H, et al: Human skeletal myotubes display a cell-autonomous circadian clock implicated in basal myokine secretion. Molecular metabolism 2015, 4:834-845.

An elegant and novel study which discovered that myokine release is regulated by a circadian clock localised in muscle.

19. Starkie R, Ostrowski SR, Jauffred S, Febbraio M, Pedersen BK: Exercise and IL-6 infusion inhibit endotoxin-induced TNF-alpha production in humans. Faseb J 2003, 17:884-886.

20. Mauer J, Chaurasia B, Goldau J, Vogt MC, Ruud J, Nguyen KD, Theurich S, Hausen AC, Schmitz J, Bronneke HS, et al: Signaling by IL-6 promotes alternative activation of macrophages to limit endotoxemia and obesity-associated resistance to insulin. Nat Immunol 2014, 15:423-430.

21. Wong PK, Campbell IK, Egan PJ, Ernst M, Wicks IP: The role of the interleukin-6 family of cytokines in inflammatory arthritis and bone turnover. Arthritis Rheum 2003, 48:1177-1189.

22. Haddad F, Zaldivar F, Cooper DM, Adams GR: IL-6-induced skeletal muscle atrophy. J Appl Physiol 2005, 98:911-917.

23. Pedersen BK, Fischer CP: Physiological roles of muscle-derived interleukin-6 in response to exercise. Curr Opin Clin Nutr Metab Care 2007, 10:265-271.

24. Febbraio MA, Hiscock N, Sacchetti M, Fischer CP, Pedersen BK: Interleukin-6 is a novel factor mediating glucose homeostasis during skeletal muscle contraction. Diabetes 2004, 53:1643-1648.

25. Calabrese LH, Rose-John S: IL-6 biology: implications for clinical targeting in rheumatic disease. Nature reviews Rheumatology 2014, 10:720-727.

26. Covington JD, Tam CS, Bajpeyi S, Galgani JE, Noland RC, Smith SR, Redman LM, Ravussin E: Myokine Expression in Muscle and Myotubes in Response to Exercise Stimulation. Med Sci Sports Exerc 2016, 48:384-390.

27. Yeo NH, Woo J, Shin KO, Park JY, Kang S: The effects of different exercise intensity on myokine and angiogenesis factors. J Sports Med Phys Fitness 2012, 52:448-454.

28. Gill N, Paltser G, Ashkar AA: Interleukin-15 expression affects homeostasis and function of B cells through NK cell-derived interferon-gamma. Cell Immunol 2009, 258:59-64.

29. McInnes IB, Leung BP, Sturrock RD, Field M, Liew FY: Interleukin-15 mediates T cell-dependent regulation of tumor necrosis factor-alpha production in rheumatoid arthritis. Nat Med 1997, 3:189-195.

30. Quinn LS, Haugk KL, Grabstein KH: Interleukin-15: a novel anabolic cytokine for skeletal muscle. Endocrinology 1995, 136:3669-3672.

31. Quinn LS, Anderson BG, Drivdahl RH, Alvarez B, Argiles JM: Overexpression of interleukin-15 induces skeletal muscle hypertrophy in vitro: implications for treatment of muscle wasting disorders. Exp Cell Res 2002, 280:55-63.

32. Nielsen AR, Mounier R, Plomgaard P, Mortensen OH, Penkowa M, Speerschneider T, Pilegaard H, Pedersen BK: Expression of interleukin-15 in human skeletal muscle effect of exercise and muscle fibre type composition. J Physiol 2007, 584:305-312.

33. Carbo N, Lopez-Soriano J, Costelli P, Alvarez B, Busquets S, Baccino FM, Quinn LS, Lopez-Soriano FJ, Argiles JM: Interleukin-15 mediates reciprocal regulation of adipose and muscle mass: a potential role in body weight control. Biochim Biophys Acta 2001, 1526:17-24.

34. Figueras M, Busquets S, Carbo N, Barreiro E, Almendro V, Argiles JM, Lopez-Soriano FJ: Interleukin15 is able to suppress the increased DNA fragmentation associated with muscle wasting in tumourbearing rats. FEBS Lett 2004, 569:201-206. 
35. Harcourt LJ, Holmes AG, Gregorevic P, Schertzer JD, Stupka N, Plant DR, Lynch GS: Interleukin-15 administration improves diaphragm muscle pathology and function in dystrophic mdx mice. Am J Pathol 2005, 166:1131-1141.

36. Pistilli EE, Alway SE: Systemic elevation of interleukin-15 in vivo promotes apoptosis in skeletal muscles of young adult and aged rats. Biochem Biophys Res Commun 2008, 373:20-24.

37. Pistilli EE, Bogdanovich S, Garton F, Yang N, Gulbin JP, Conner JD, Anderson BG, Quinn LS, North K, Ahima RS, Khurana TS: Loss of IL-15 receptor alpha alters the endurance, fatigability, and metabolic characteristics of mouse fast skeletal muscles. J Clin Invest 2011, 121:3120-3132.

38. Henningsen J, Pedersen BK, Kratchmarova I: Quantitative analysis of the secretion of the MCP family of chemokines by muscle cells. Mol Biosyst 2011, 7:311-321.

39. Lightfoot AP, Goljanek-Whysall, K, Earl, K.E, Cotton, C.V, McArdle, A, Cooper, R.G: Is Muscle a Chemotactic Organ in the Idiopathic Inflammatory Myopathies (IIM)? Overexpression of MHC I (H$2 \mathrm{~Kb}$ ) in $\mathrm{C} 2 \mathrm{C} 12$ Myotubes Results in Release of Pro-Inflammatory Cytokines. Rheumatology (Oxford) 2015, 54:i43.

40. Warren GL, Hulderman T, Mishra D, Gao X, Millecchia L, O'Farrell L, Kuziel WA, Simeonova PP: Chemokine receptor CCR2 involvement in skeletal muscle regeneration. Faseb J 2005, 19:413-415.

• 41. Kanzleiter T, Rath M, Gorgens SW, Jensen J, Tangen DS, Kolnes AJ, Kolnes KJ, Lee S, Eckel J, Schurmann A, Eckardt K: The myokine decorin is regulated by contraction and involved in muscle hypertrophy. Biochem Biophys Res Commun 2014, 450:1089-1094.

This study identified decorin as a novel exercise-induced myokine and characterised its hypertrophic function, i.e binding to and thus inhibitingmyostatin.

••42. Amato AA, Sivakumar K, Goyal N, David WS, Salajegheh M, Praestgaard J, Lach-Trifilieff E, Trendelenburg AU, Laurent D, Glass DJ, et al: Treatment of sporadic inclusion body myositis with bimagrumab. Neurology 2014, 83:2239-2246.

A novel clinical study using an activin type II receptor antagonist to inhibit myostatin activity and thus promote muscle hypertrophy.

43. Nagaraju K, Casciola-Rosen L, Lundberg I, Rawat R, Cutting S, Thapliyal R, Chang J, Dwivedi S, Mitsak M, Chen YW, et al: Activation of the endoplasmic reticulum stress response in autoimmune myositis: potential role in muscle fiber damage and dysfunction. Arthritis Rheum 2005, 52:1824-1835.

44. Lightfoot AP, Nagaraju, K, McArdle, A, Cooper R.G: Understanding the origin of non immune-cell mediated weakness in the idiopathic inflammatory myopathies (IIM) - Potential role of ER stresspathways. Curr Opin Rheumatol 2015, 27:580-585.

45. Welc SS, Judge AR, Clanton TL: Skeletal muscle interleukin-6 regulation in hyperthermia. Am J Physiol Cell Physiol 2013, 305:C406-413.

•46. Kim S, Joe Y, Kim HJ, Kim YS, Jeong SO, Pae HO, Ryter SW, Surh YJ, Chung HT: Endoplasmic reticulum stress-induced IRE1alpha activation mediates cross-talk of GSK-3beta and XBP-1 to regulate inflammatory cytokine production. J Immunol 2015, 194:4498-4506.

This study identified that cytokine expresion and release is regulated by key components of the ER stress pathway.

47. Coley W, Rayavarapu S, Pandey GS, Sabina RL, Van der Meulen JH, Ampong B, Wortmann RL, Rawat R, Nagaraju K: The molecular basis of skeletal muscle weakness in a mouse model of inflammatory myopathy. Arthritis Rheum 2012, 64:3750-3759.

•48. Alemo-Munters L, Loell I, Ossipova E, Raouf J, Dastmalchi M, Lindroos E, Chen Y-W, Esbjörnsson M, Korotkova M, Alexaderson H, Nagaraju K, Crofford LJ, Jakobsson P-J, Lundberg IE. Endurance exercise improves molecular pathways of aerobic metabolism in patients with myositis. Arthritis Rheum 2016, 68(7):1738-50.

First study to describe beneficial molecular changes in the muscle of myositis patients following endurance exercise.

49. Benatti FB, Pedersen BK: Exercise as an anti-inflammatory therapy for rheumatic diseases-myokine regulation. Nature reviews Rheumatology 2015, 11:86-97.

Legend to Figure 1: Schematic summary of current knowledge regarding myokine generation and paracrine release from skeletal muscle ( $\rightarrow$ means paracrine activation while $\dashv$ means paracrine inhibition) in response to exercise and/or inflammatory stimuli. Tumour necrosis factor alpha (TNF $\alpha$ ) induces myokine release from 
muscle, mediated by increased generation of reactive oxygen species (ROS) and by nuclear factor kappa B (NFkB) activation, which are attenuated in the presence of antioxidants, e.g. $N$-acetylcysteine (NAC) and SS-31. Exercise induces upregulation in gene expression and release of IL-6, IL-8, IL-15 and decorin, the latter binding to and inhibiting myostatin. Exercise-induced IL-6 provokes metabolic changes in muscle, via increased glucose uptake and fatty acid (FA) oxidation. Muscle-derived IL-8 is associated with pro-angiogenesis, while IL-15 promotes muscle hypertrophy. Exercise-induced downregulation of myostatin, itself a negative regulator of muscle mass, is reported to mediate hypertrophic adaptive responses. Similarly, when decorinis released from muscle following exercise, it binds to and thus inhibits myostatin, inducing a muscle hypertrophic response. Pharmacological targeting of the myostatin receptor (i.e. activin type II) with the biological agent Bimagrumab, has been successfully used in IBM to competitively inhibit the action of myostatin as a means of promoting therapeutic muscle hypertrophy [42]. 